\title{
Mental health, serum biomarkers and survival in severe COPD: a pilot study
}

\author{
Christian Zilz ${ }^{1 *}$, Stefan H. Blaas ${ }^{2}$, Michael Pfeifer ${ }^{2,4}$, Rudolf A. Jörres ${ }^{3}$ and Stephan Budweiser ${ }^{5}$
}

\begin{abstract}
Background: Chronic obstructive pulmonary disease (COPD) impairs physical status and impacts on mental health. This prospective study was designed to assess associations between mental health and systemic biomarkers, and their combined relationship with long-term survival in stable severe COPD.

Methods: Forty-five patients with severe but stable COPD (forced expiratory volume in $1 \mathrm{~s}$ of 29.8 (quartiles: 22.6; 41.4) \%predicted) were assessed using the Hospital Anxiety and Depression Scale (HADS), the Patient Health Questionnaire (PHQ), St. George's Respiratory Questionnaire and the State-Trait Anxiety Inventory (STAI). The following serum biomarkers were measured: $25-\mathrm{OH}$-cholecalciferol, C-reactive protein, erythrocyte sedimentation rate, leucocyte number, serum amyloid-A (SA-A), N-terminal pro-brain natriuretic peptide, troponin I, glycosylated haemoglobin, haemoglobin $(\mathrm{Hb})$, haematocrit $(\mathrm{Hc})$, creatinine and thyroid-stimulating hormone. Patients were followed-up for 36 months. Associations between aspects of mental health and biomarkers, and their utility as predictors of 3-year survival were evaluated by regression analyses.
\end{abstract}

Results: The prevalence of anxiety (HADS-A: $89.9 \%$ ), depression (HADS-D: $58.8 \%$; PHQ: $60.6 \%$ ), somatisation (PHQ-15: $81.8 \%$ ) and psychosocial stress (PHQ-stress: $60.6 \%$ ) was high. There was a significant positive association between the leucocyte count and SA-A level with STAl-trait anxiety ( $p=0.03$ and $p=0.005$, respectively), and between leucocytes and PHQ-stress $(p=0.043)$. $\mathrm{Hb}$ and Hc were significantly negatively associated with HADS-depression ( $p=0.041$ and $p=0.031$, respectively). Univariate Cox regression analyses revealed that leucocyte count (hazard ratio (HR) 2.976, $95 \% \mathrm{Cl} 1.059-8.358 ; p=0.038$ ), and stress (HR 4.922, $95 \% \mathrm{Cl} 1.06-22.848 ; p=0.042$ ) were linked to long-term survival. In multivariate Cox regression analyses, including known risk factors for survival in COPD, PHQ-stress (HR 45.63, $95 \%$ Cl 1.72-1,208.48; $p=0.022$ ) remained significantly associated with survival.

Conclusion: In this pilot study different dimensions of mental health were correlated to serum biomarkers, probably reflecting systemic effects of COPD. While leucocyte number and PHQ-stress were associated with long-term survival in univariate analyses, PHQ-stress remained in multivariate analyses as independent prognostic factor.

Keywords: Anxiety, Chronic respiratory disease, Depression, Mortality, Prognostic factors, Systemic inflammation

\section{Background}

Chronic obstructive pulmonary disease (COPD) is a systemic disease whose multiple dimensions are increasingly uncovered by research. This has promoted the concept of clinical phenotypes, which attempts to identify specific subgroups of COPD patients with similar clinical characteristics, treatment needs and prognosis [1]. As a result, several clinical phenotypes, which can occur simultaneously, are assumed, e.g., patients with marked

\footnotetext{
* Correspondence: christianzilz@arcor.de

'Department of Internal Medicine, HELIOS Hospital Munich-Perlach, Munich, Germany

Full list of author information is available at the end of the article
}

hyperinflation, frequent exacerbations, rapid $\mathrm{FEV}_{1}$ decline, metabolic and cardiovascular comorbidities, or eosinophilic inflammation [1].

Importantly, COPD not only impairs physical status but also has impacts on mental health [2-5]. Owing to the prevalence of mental health problems in COPD further evaluation is needed. Mental health involves various aspects, such as psychiatric issues, somatisation and disease-evoked psychological strain. Among these, depression and anxiety are strongly related to worse long-term outcome in COPD [6-8]. 
Similarly, health-related quality of life (HRQOL) is a broad multidimensional concept that usually includes self-reported measures of physical and mental health [9], and is also associated with long-term survival $[10,11]$ and acute exacerbation [12].

Unfortunately, assessing mental health is time consuming and complex, because either an interview with an experienced expert or complicated psychosocial assessments with questionnaires are necessary. Further problems lie in the quality of the completed questionnaires. Patients may have a poor compliance with regard to mental health assessment via questionnaires, perhaps due to misunderstanding, age-related diseases (e. g., cognitive or vision impairment) or lack of interest. Clinically desirable would be an alternative approach in assessing mental health, especially if it is independent from the patient's active cooperation, as well as easily to obtain.

Serum biomarkers have been mostly explored in COPD for estimating the risks of exacerbation and for long-term monitoring [13], including procalcitonin [14], N-terminal pro-brain natriuretic peptide (NT-ProBNP) and troponin [15] for short-term assessment, and leukocyte number, fibrinogen and CRP in relation to long-term survival [16], as well as blood eosinophil count as biomarker for the response to inhaled steroids [17].

Due to its systemic involvement, associations with serum biomarkers could also occur regarding mental health, particularly in severe COPD. Indeed, associations between depression and the level of biomarkers, such as C-reactive protein (CRP) [18], haemoglobin (Hb) [19], thyroid-stimulating hormone (TSH) $[20,21]$ and vitamin D [22-25], have been reported.

Based on these considerations, it seems plausible to explore possible associations between mental health parameters and serum biomarkers. We therefore assessed whether in stable severe COPD different dimensions of mental health assessed via questionnaires correlated with established serum biomarkers and whether they were associated with long-term survival.

\section{Methods}

\section{Study population}

This prospective study included patients with COPD Global Initiative for Chronic Obstructive Lung Disease (GOLD) stages III and IV, aged 35-95 years, admitted to the Center for Pneumology at Donaustauf Hospital in a clinically stable state between October 2009 and October 2010. Patients with a moderate to severe exacerbation defined by a serum CRP level $\geq 20 \mathrm{mg} / \mathrm{dl}$, a blood $\mathrm{pH}<7.35$, or having received antibiotic therapy within the last 2 weeks were not included. Other exclusion criteria were acute pneumonia confirmed by X-ray; lung resection; thoracic deformations, or refusal to participate.
Eligible patients were enrolled at control appointments for non-invasive intermittent positive pressure ventilation or long-term oxygen therapy, and from new referrals to the clinic. Demographic data, blood samples, physical function, mMRC dyspnea scale, Cumulative Illness Rating Scale-Geriatric and mental health (questionnaires) were assessed after inclusion in the study; notification of death during the study period (at least 36 months for each patient after inclusion) was obtained from the patient's relatives or family doctor by telephone.

\section{Blood samples}

Peripheral blood samples were evaluated for the following: leucocyte count, $\mathrm{Hb}$ level and the haematocrit $(\mathrm{Hc})$ (ABX Micros 60-CT analyser, equipped with v.1.0 software, Horiba ABX, Montpellier, France); creatinine, CRP and TSH levels (Dimension Xpand system, Dade Behring, Schwalbach, Germany); glycosylated haemoglobin (HbA1c; Cobas Integra 400 plus analyser, Roche Diagnostics Deutschland $\mathrm{GmbH}$, Mannheim, Germany); 25-OHcholecalciferol (25-OHD), serum amyloid-A (SA-A) and troponin I (ADVIA Centaur TnI-Ultra assays, Bayer Vital GmbH, Fernwald); NT-proBNP (Elecsys 2010 analyser, Roche Diagnostics $\mathrm{GmbH}$ ); and the erythrocyte sedimentation rate (ESR).

\section{Functional analysis}

Lung function assessment comprised post-bronchodilator spirometry and body plethysmography performed using a Masterlab system (Viasys Inc., Würzburg, Germany) according to the recommendations of the American Thoracic Society (ATS) [26]. The predicted values for forced expiratory volume in $1 \mathrm{~s}\left(\mathrm{FEV}_{1}\right)$ and vital capacity (VC) were calculated using the reference values determined by the European Community for Steel and Coal [27].

A six-minute walk distance (6-MWD) test was performed according to the recommendations of the ATS [28]. The predicted 6-MWD was calculated using age, gender, height and weight [29].

\section{Mental health}

Aspects of mental health were measured using the German version of the Hospital Anxiety and Depression Scale (HADS) [30], the Patient Health Questionnaire (PHQ) [31], the St. George's Respiratory Questionnaire (SGRQ) [32] and the State-Trait Anxiety Inventory (STAI) [33].

The HADS was originally developed as a screening tool for anxiety and depression in an outpatient setting but is now widely used in general hospital practice for non-psychiatric clinical populations [30, 34]. It is composed of a total of 14 questions on a questionnaire each scored on a four-point Likert scale (0-3); there are seven questions for symptoms of anxiety (HADS-A) and seven 
for symptoms of depression (HADS-D) each, therefore, with a total score range of $0-21$ [30]. Higher scores indicate more severe symptoms [30]. The following categories (cut-off scores) for HADS-A and HADS-D were used: no (0-7), borderline (8-10), severe (11-14) and very severe $(15-21)$.

The PHQ is a self-administered version of the Primary Care Evaluation of Mental Disorders (PRIME-MD) and is used in primary medical practice to determine psychosocial and somatic stress factors as well as to screen for the five most frequent psychiatric disease groups [31, 35]. The complete version comprises 78 questions to which answers are evaluated with two-point to five-point Likert scales, and one open question [31, 35]. In the present study the modules of depression (PHQ-9) with nine items (score range 0-27), somatic factors (PHQ-15) with 15 items (score range $0-30$ ) and stress (PHQ-stress) with ten items (score range $0-20$ ) were used [36, 37]. Higher scores indicate more severe symptoms [35-37].

As categories (cut-off scores) were used for PHQ-9: no-minimal (0-4), mild (5-9), moderate (10-14) moderate to severe (15-19) and severe (20-27); for PHQ-15: no-minimal (0-4), low (5-9), medium (10-14) and high (15-30), and for PHQ-stress: no-minimal (0-4), mild (5-9), moderate (10-14) and severe (15-20). Depression, somatisation and psychosocial stress were assumed for scores $\geq 5$.

The SGRQ was designed to measure disease-specific health impairment in patients with chronic airflow limitation. It consists of two parts and measures three components (symptoms, activity and impact) and a total score each with a score range of 0-100. Higher scores indicate more limitations [32].

The STAI was developed to reveal state and trait anxiety and is used in clinical and research settings. Two forms are used, (X1-state (STAI-state) anxiety and X2-trait (STAI-trait) anxiety), with 20 items each and a four-point Likert scale. The scores can range from 20 to 80; higher scores mean more anxiety [33].

\section{Study approval}

The study was approved by the ethics review committee of the University of Regensburg, Germany (approval number 09/097). Written informed consent was obtained from the patients.

\section{Statistical analysis}

Continuous variables are shown as median values and quartiles. Spearman's rank correlation coefficient was used to identify and measure the strength of the relationship between biomarkers. Associations between biomarkers and mental health were analysed using univariate linear regression analyses, with mental health as a dependent variable. Independent associations were assessed in an adjusted multivariate linear analysis (method: enter). Survival (until death from any cause) was analysed using the Mann-Whitney $U$ test, Cox proportional hazard regression (univariate and, if applicable, multivariate) analyses, and Kaplan-Meier analyses (logrank test), starting by the day of inclusion and ending after 3-years of follow up. As cut-off we used median values. Variables significant in univariate Cox proportional hazard regression analyses and known risk factors for survival in COPD were entered into multivariate Cox proportional hazard regression analyses (method: stepwise backward likelihood ratio; probability for stepwise entry/removal: $0.05 / 0.10$ ) to identify independent predictors. $\mathrm{P}<0.05$ were considered statistically significant. SPSS version 20.0 (IBM SPSS Statistics, USA) was used for the data analysis.

\section{Results}

\section{Study population}

At the screening 279 patients out of 621 (44.9\%) showed GOLD stage III/IV. Among the 279 COPD GOLD stage III/IV patients, 106 (38 \%) had no interest in participating, 86 (30.8 \%) showed characteristics leading to exclusion (exacerbations, acute pneumonia, antibiotic therapy in the last 2 weeks, lung resection, thoracic deformations, or neuromuscular disease), and 42 (15.1\%) did not come to a scheduled visit (Fig. 1). Forty-five patients were eligible for inclusion in the study, and their characteristics are shown in Tables 1 and 2. No significant differences in patient characteristics were observed with respect to gender, with the exception of nicotine consumption, which was lower in females $(32(30 ; 50)$ vs. $53(40 ; 80)$ pack/years; $p=0.024$ ).

\section{Mental health}

Not all patients completed the questionnaires sufficiently (completed questionnaires: HADS, $n=34$; PHQ, $n=33$; STAI, $n=33$; and SGRQ, $n=35$ ). However, no significant differences were detected between patients who did or did not. Furthermore, no significant differences in mental health were found with regard to gender. The results of all questionnaires are shown in Table 2.

\section{HADS: anxiety and depression}

Anxiety, demonstrated by the HADS-A score, was present in 28 patients out of 34 (89.9\%); 13 patients out of 34 (38.2\%) showed borderline, 12 (35.3\%) severe, and 3 $(8.8 \%)$ very severe anxiety (Table 2 , Fig. 2 ).

The prevalence of depression, demonstrated by the HADS-D score, was 20 patients out of 34 (58.8\%); 10 patients out of 34 (29.4\%) had borderline, 8 (23.5\%) severe, and 2 (5.9\%) had very severe depression. 


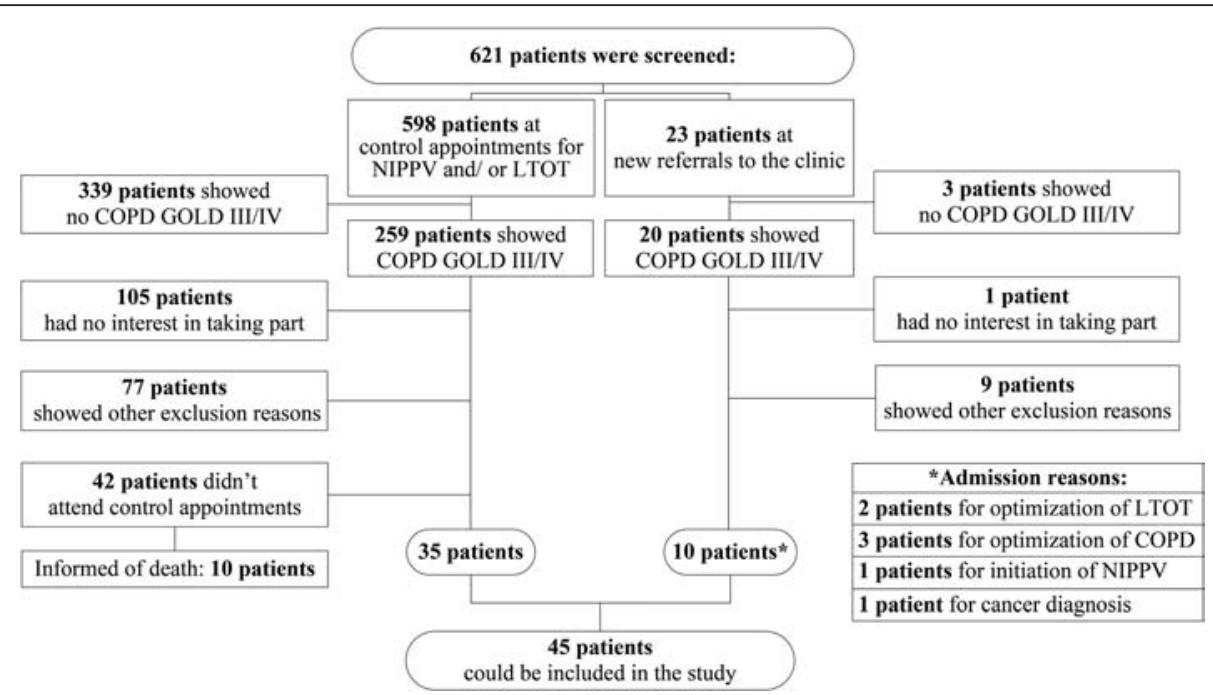

Fig. 1 Patients' recruitment. Notes: Other exclusion reasons: acute pneumonia confirmed by X-ray; lung resection; thoracic deformations; C-reactive protein (CRP) $\geq 20 \mathrm{mg} / \mathrm{dl}$; blood $\mathrm{pH}<7.35$; antibiotic therapy within the last 2 weeks. Abbreviations: NIPPV, non-invasive intermittent positive pressure ventilation; LTOT, long-term oxygen therapy

\section{PHQ: depression, somatisation and stress}

The scores of PHQ-9 (Fig. 3) indicated a depression rate of 20 patients out of $33(60.6 \%)$. Mild depression was apparent in 11 patients out of 33 (33.3\%); moderate in 3 (9.1\%); moderate to severe in $5(15.2 \%)$ and severe depression in 1 (3\%). The somatisation rate, as demonstrated by PHQ-15 scores, was 27 patients out of 33 $(81.8 \%)$, with low, medium and high somatic symptoms split equally 9 patients out of $33(27.3 \%)$. The mild to severe stress rate, as demonstrated by PHQ-stress scores, was 20 patients out of $33(60.6 \%)$ with 14 patients out of $33(42.4 \%)$ mild cases, 5 (15.2 \%) moderate and $1(3 \%)$ severe.

\section{SGRQ and STAI}

Due to the lack of commonly established cut-off values for SGRQ and STAI, only their median values are given in Table 2.

\section{Biomarkers}

The results are presented in Table 2. SA-A was the only biomarker showing differences between genders. Female $(n=14)$ patients had higher values than male $(n=29)$ patients $(16.7(7.5 ; 61.4) \mathrm{mg} / \mathrm{l}$ vs. $7.3(3.6 ; 9.0) \mathrm{mg} / \mathrm{l} ; p=$ 0.039). Correlations between biomarkers are demonstrated in Tables 3 and 4.

\section{Associations between mental health and serum biomarkers}

In univariate linear regression analyses, there were inverse associations between depression, demonstrated by the HADS-D score, and the values for $\mathrm{Hb}(p=0.041)$ or the Hc $(p=0.031)$. Positive associations were found between the PHQ-stress score and leucocyte numbers $(p=0.043)$, as well as between the STAI-trait anxiety score and leucocyte numbers $(p=0.03)$ or SA-A $(p=$ 0.005 ) (Table 5). Because the Spearman's rank correlation was high between the significant biomarkers, they were not included in a multivariate regression analysis.

\section{Associations between mental health and functional indices}

Inverse associations were demonstrated between the HADS-A score and VC $(p=0.01)$ and $\mathrm{FEV}_{1}(p=0.015)$, and between STAI-state anxiety and VC $(p=0.041)$. Furthermore, positive associations were found between the HADS-A score and residual volume/ total lung capacity $(p=0.007)$. The 6 -MWD score was negatively linked to several aspects of mental health: HADS-A ( $p=$ $0.002)$, HADS-D $(p=0.031)$, somatisation demonstrated by the PHQ-15 score $(p=0.021)$ and quality of life demonstrated by the total score of the SGRQ $(p=0.003)$. There was also an inverse association between the HADS-A score and age $(p=0.045)$ (Table 5). In multivariate linear regression analyses including significant biomarkers and functional indices, the 6-MWD score remained significant for HADS-A $(p \leq 0.024)$ and HADS-D $(p=0.042)$ (Table 6).

\section{Survival}

Each patient was observed for 3 years. Within this period 18 patients out of $45(40 \%)$ died; 9 patients $(50 \%)$ died of respiratory, 2 (11.1\%) of non-respiratory, and 7 (38.9\%) of not further specified causes.

The Mann-Whitney- $U$ test did not demonstrate any significant differences in the tested variables between 
Table 1 Patient's characteristics $(n=45)$

\begin{tabular}{|c|c|}
\hline \multicolumn{2}{|l|}{ Demographics } \\
\hline Age, years & $64(57 ; 72)$ \\
\hline Male & $31(68.9)$ \\
\hline Female & $14(31.1)$ \\
\hline $\mathrm{BMI}, \mathrm{kg} / \mathrm{m}^{2}$ & $28.1(6.8)$ \\
\hline \multicolumn{2}{|l|}{ Smoking history } \\
\hline Active tobacco exposure, pack/years & $50(30 ; 70)$ \\
\hline Current smoker & $6(13.3)$ \\
\hline Ex-smoker & $38(84.4)$ \\
\hline Never smoker & $1(2.2)$ \\
\hline \multicolumn{2}{|l|}{ Spirometery } \\
\hline VC, \% predicted & $61.8(50 ; 71.4)$ \\
\hline $\mathrm{FEV}_{1}, \%$ predicted & $29.8(22.6 ; 41.4)$ \\
\hline $\mathrm{FEV}_{1} / \mathrm{FVC}, \%$ & $45.5(39 ; 53.3)$ \\
\hline \multicolumn{2}{|l|}{ Blood gas analysis $^{a}$} \\
\hline $\mathrm{paO}_{2}, \mathrm{mmHg}$ & $62(52.5 ; 73)$ \\
\hline $\mathrm{paCO}_{2}, \mathrm{mmHg}$ & $44(40 ; 53.5)$ \\
\hline $\mathrm{BE},(\mathrm{mmol} / \mathrm{l})$ & $3.6(1.45 ; 6.55)$ \\
\hline $\mathrm{pH}$ & $7.41(7.39 ; 7.44)$ \\
\hline \multicolumn{2}{|l|}{ mMRC dyspnea scale $e^{b}$} \\
\hline Grade 0 & $3(8.1)$ \\
\hline Grade 1 & $1(2.7)$ \\
\hline Grade 2 & $5(13.5)$ \\
\hline Grade 3 & $9(24.3)$ \\
\hline Grade 4 & $19(51.4)$ \\
\hline \multicolumn{2}{|l|}{ Comorbidity- CIRS-G } \\
\hline Total score ${ }^{c}(0-56)$ & $13(10 ; 17)$ \\
\hline Severity index ${ }^{d}(0-14)$ & $2.4(2.2 ; 2.7)$ \\
\hline \multicolumn{2}{|l|}{ NIPPV/LTOT } \\
\hline NIPPV & $35(77.8)$ \\
\hline LTOT & $41(91.1)$ \\
\hline LTOT and NIPPV & $33(73.3)$ \\
\hline Only LTOT & $8(17.8)$ \\
\hline Only NIPPV & $2(4.4)$ \\
\hline \multicolumn{2}{|l|}{ Medications (selection) } \\
\hline Systemic corticosteroids ${ }^{\mathrm{e}}$ & $15(33.3)$ \\
\hline Inhaled corticosteroids & $37(82.2)$ \\
\hline LABA & $37(82.3)$ \\
\hline SAMA & $40(88.9)$ \\
\hline SABA and/or SAMA & $31(68.9)$ \\
\hline Theophylline & $18(40)$ \\
\hline
\end{tabular}

Table 1 Patient's characteristics $(n=45)$ (Continued)

\begin{tabular}{|c|c|}
\hline Anxiolytics and/or antidepressants & $19(42.2)$ \\
\hline Statins & $13(28.9)$ \\
\hline prosis prophylaxis & 7.8) \\
\hline \multicolumn{2}{|c|}{ 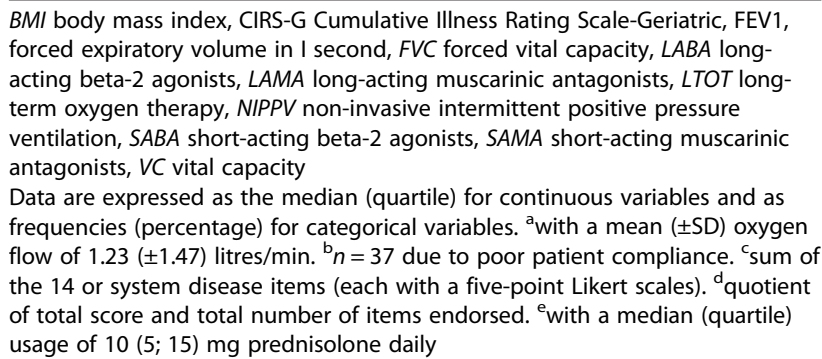 } \\
\hline
\end{tabular}

survivors and non-survivors. However, using the median as cut-off in a Cox proportional hazard regression model, leucocyte numbers (hazard ratio (HR) 2.97, $95 \%$ CI 1.06-8.36; $p=0.038$ ) and PHQ-stress (HR 4.92, $95 \%$ CI $1.06-22.85 ; p=0.042)$ were significantly linked to long-term survival (Table 7 and Fig. 4). In multivariate Cox regression analyses including known risk factors for survival in COPD (6-MWD, body mass index, $\mathrm{FEV}_{1}$ (\% predicted)) as well as PHQ-stress (model 1) and leucocytes (model 2), only PHQ-stress (HR 45.63, $95 \%$ CI $1.72-1,208.48 ; p=0.022$ ) remained significantly related to survival, whereas leucocyte numbers did not (Table 7 , model 1 and 2).

\section{Discussion}

In this prospective study of patients with stable COPD of GOLD stages III and IV we have examined the association between mental health measures and serum biomarkers by various statistical methods. Biomarkers and mental health were also analysed for associations with survival. There were statistically significant but rather weak associations between some aspects of mental health and $\mathrm{SA}-\mathrm{A}, \mathrm{Hb}, \mathrm{Hc}$ and leucocyte number. Among the mental health parameters analysed PHQ-stress and among the biomarkers only leucocyte number were associated with survival, whereas in an adjusted multivariate analysis only PHQ-stress remained as independent prognostic factor.

\section{Mental health}

We found that the prevalence of anxiety (HADS-A: $84.9 \%$ ) and depression (HADS-D: $58.8 \%$; PHQ-9: $60.6 \%)$ was higher than reported in studies that included patients in primary care with moderate to very severe COPD of comparable age (Centers for Epidemiologic Studies Depression scale (severe COPD): 25 \% [38]; HADS-A (cut-off $\geq 11$; moderate to very severe COPD): $32.7 \%$, HADS-D: (cut-off $\geq 11$; moderate to very severe COPD): $20.8 \%$ [39]). This was probably due to the 
Table 2 Characteristics of questionnaires and serological biomarkers

\begin{tabular}{|c|c|c|}
\hline Explanatory Variables & $\mathrm{n}$ & Median (quartiles) \\
\hline \multicolumn{3}{|l|}{ Questionnaires: } \\
\hline \multicolumn{3}{|l|}{ HADS (0-21) } \\
\hline Anxiety & 34 & $10(8.75 ; 11)$ \\
\hline Depression & 34 & $8.5(4 ; 11.3)$ \\
\hline \multicolumn{3}{|l|}{ PHQ } \\
\hline Depression (0-27) & 33 & $6(3 ; 11)$ \\
\hline Somatisation (0-30) & 33 & $10(6 ; 15)$ \\
\hline Stress (0-20) & 33 & $6(3 ; 8)$ \\
\hline \multicolumn{3}{|l|}{ SGRQ (0-100) } \\
\hline Total score & 35 & $71.3(57.5 ; 78.2)$ \\
\hline Activity & 35 & $85.9(76.1 ; 92.5)$ \\
\hline Impact & 35 & $63.7(42.5 ; 71.1)$ \\
\hline Symptoms & 35 & $69.2(49 ; 76)$ \\
\hline \multicolumn{3}{|l|}{ STAI (20-80) } \\
\hline State anxiety & 33 & $45(34,5 ; 54)$ \\
\hline Trait anxiety & 33 & $45(36 ; 49,5)$ \\
\hline \multicolumn{3}{|l|}{ Biomarkers: } \\
\hline \multicolumn{3}{|l|}{ Systemic inflammation: } \\
\hline 25-OHD (nmol/l) & 44 & $38.4(25.3 ; 66.5)$ \\
\hline CRP $(\mathrm{mg} / \mathrm{dl})$ & 45 & $2.6(1.1 ; 8.7)$ \\
\hline $\operatorname{ESR}(\mathrm{mm} / \mathrm{h})$ & 33 & $17(7.5 ; 40)$ \\
\hline Leucocytes $\left(10^{3} / \mu l\right)$ & 45 & $9.7(7.4 ; 12.4)$ \\
\hline SA-A (mg/l) & 43 & $8.1(4 ; 17.8)$ \\
\hline \multicolumn{3}{|l|}{ Cardiac dysfunction: } \\
\hline NT-proBNP (pg/ml) & 45 & $176(65 ; 300)$ \\
\hline Troponin I (ng/ml) & 45 & $0.013(0.007 ; 0.02)$ \\
\hline \multicolumn{3}{|l|}{ Other: } \\
\hline HbAlc (\%) & 45 & $6.2(5.8 ; 6.9)$ \\
\hline $\mathrm{Hb}(\mathrm{g} / \mathrm{dl})$ & 45 & $13.8(13 ; 15.2)$ \\
\hline Hc (\%) & 45 & $43(40 ; 47)$ \\
\hline Creatinine (mg/dl) & 45 & $1.0(0.8 ; 1.3)$ \\
\hline TSH (mU/l) & 45 & $0.8(0.5 ; 1.2)$ \\
\hline
\end{tabular}

HADS cut-off scores: no (0-7), borderline (8-10), severe (11-14) and very severe (15-21) anxiety (HADS-anxiety) or depression (HADS-depression). PHQ-9 cut-off scores: no-minimal (0-4), mild (5-9), moderate (10-14) moderate to severe (15-19) and severe (20-27) depression. PHQ-15 cut-off scores: no-minimal (0-4), low (5-9), medium (10-14) and high (15-30) somatic factors. PHQ-stress cut-off scores: no-minimal (0-4), mild (5-9), moderate (10-14) and severe (15-20) psychosocial stress. For PHQ: Depression, somatisation and psychosocial stress were assumed for scores $\geq 5$. SGRQ and STAI: no commonly established cut-off values. For all questionnaires: Higher scores indicate more limitations or more severe symptoms

25-OHD 25-OH-cholecalciferol, CRP C-reactive protein, ESR erythrocyte sedimentation rate, HADS Hospital Anxiety and Depression Scale, HbAlc glycosylated haemoglobin, $\mathrm{Hb}$ haemoglobin, Hc haematocrit, NT-proBNP N-terminal pro-brain natriuretic peptide, $P H Q$ Patient Health Questionnaire,

SA-A serum amyloid-A, SGRQ St. George's Respiratory Questionnaire, STAI State-Trait Anxiety Inventory, TSH thyroid-stimulating hormone selection, in our study, of patients with severe and very severe COPD, and the inclusion of patients with borderline anxiety and depression (HADS). Moreover, there were also differences in the questionnaires used. When we applied to our cohort the same HADS cut-off value as used in previous reports (cut-off $\geq 11$ ), the prevalence of anxiety (46.7 \%) and depression (29.4\%) was still higher. The exclusion of patients categorised with mild depression in the PHQ-9 (cut-off $\geq 10$ ) gave a similar depression rate of $27.3 \%$. The frequency of somatisation (PHQ-15: 81.8 \%) was also high in our study participants in comparison to the general population (PHQ-15: $9.3 \%$ [40]. The stress score was also higher compared to primary care patients of similar age (PHQ-stress score: 6 vs. 2.3) [41] and showed an increased stress rate (cut-off: $\geq 5$ ) with $60.6 \%$. These results highlight the importance of mental health and psychiatric diagnoses particularly in patients with severe COPD, including the view that COPD has to be recognized as systemic disease that obviously has tremendous effects beyond the somatic dimension.

\section{Mental health and biomarkers}

In our study, leucocyte number and the level of SA-A, biomarkers particularly related to systemic inflammation, were significantly correlated with mental health. In line with this finding, Justo and co-workers described associations between a history of mental crisis and inflammatory markers (fibrinogen and leucocyte number) in men [42]. Other investigators have reported associations between inflammation (defined by increased levels of cytokines and CRP) and neuropsychiatric disease, such as major depressive disease [43], generalised anxiety disorder [44], or even anxiety in healthy adults [45]. In accordance with this, Thompsen et al. demonstrated that elevated leukocytes levels are associated with a higher risk for comorbidities in COPD [46]. Prolonged neuroinflammation, induced by pro-inflammatory cytokines, is considered to play an important role in the development of neurobehavioral impairment, dementia and cognitive decline [29]. The inflamed brain microenvironment apparently leads to an overactivation of microglial cells with increased release of reactive oxygen species that cause neurotoxicity [47], and to dysfunction of central nervous system stem cells with resulting impairment in tissue homeostasis and repair function [48].

These hypotheses has been recently supported by the findings of Al-shair et al. [49] who described a strong positive correlation between tumor necrosis factor-alpha (TNF- $\alpha$ ) and depression - measured by the Brief Assessment Schedule Depression Cards (BASDEC) - in mainly moderate and stable COPD patients [49]. TNF- $\alpha$ is an inflammatory protein that has been shown to be increased in stable COPD (compared to healthy control 

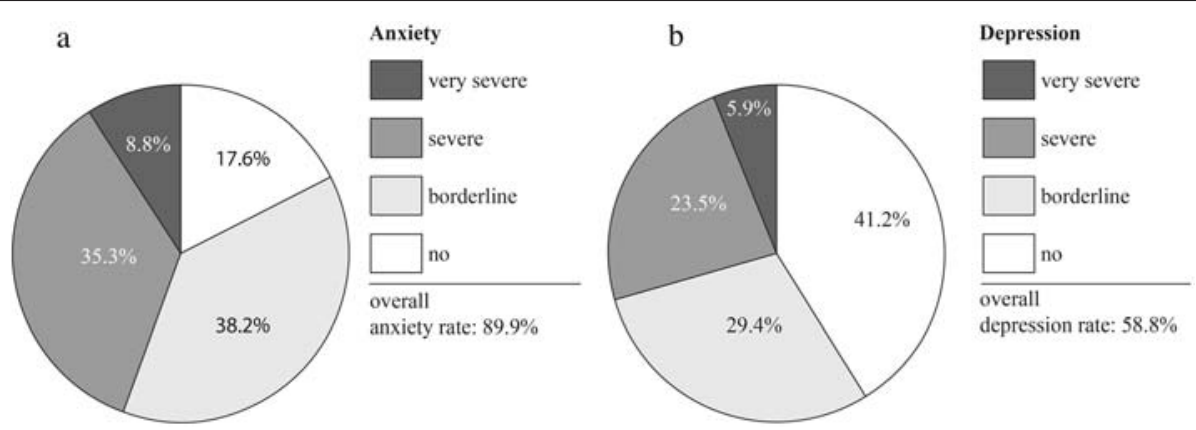

Fig. 2 Hospital Anxiety (a) and Depression (b) Scale (HADS)
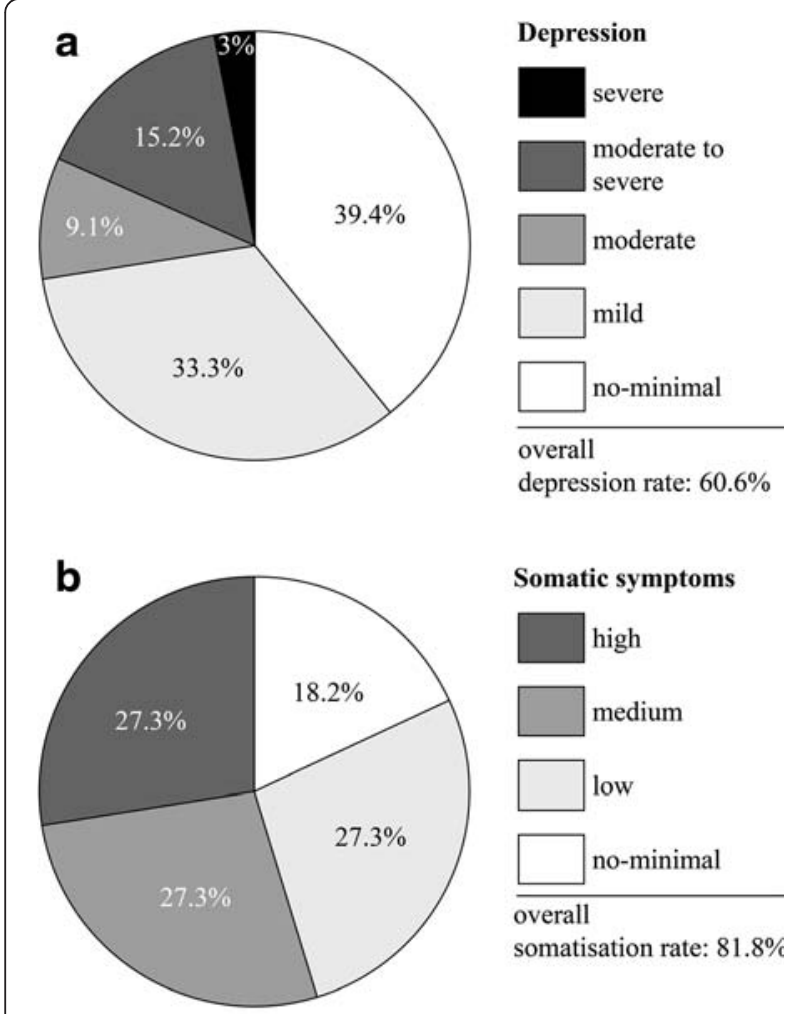

Somatic symptoms
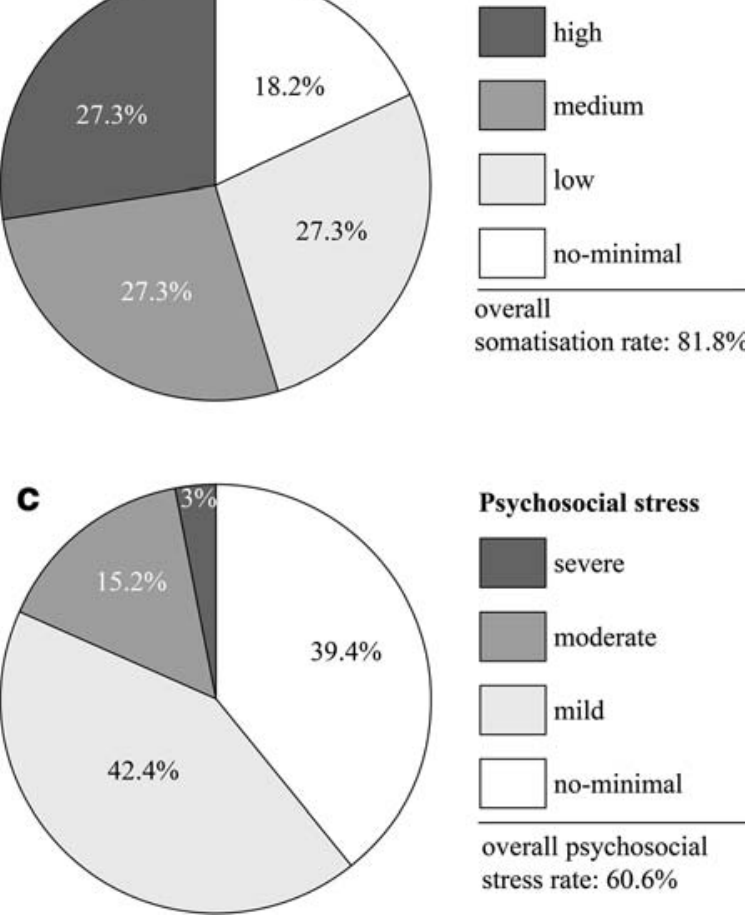

Psychosocial stress

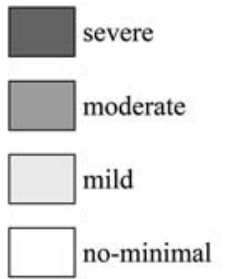

overall psychosocial stress rate: $60.6 \%$

Fig. 3 Patient Health Questionnaire (PHQ): scores of depression (a), somatic symptoms (b) and psychosocial stress (c)

groups) and seems to increase even more in acute exacerbation of COPD [50]. As inflammatory mediator, besides others, it induces acute phase proteins including SA-A, which has been shown to be a more sensitive marker of an acute exacerbation of COPD than CRP alone or with dyspnea [51]. According to the present study, SA-A seems to be also a sensitive marker for negative effects on mental health in patients with stable and severe COPD.

In addition, in our study lower $\mathrm{Hb}$ and $\mathrm{Hc}$ levels were linked to higher depression values. Accordingly, we found in a large retrospective analysis that in patients with chronic respiratory failure, irrespective of the underlying aetiology, anaemia was independently related to dyspnoea and to a low HRQOL score when measured by the Severe Respiratory Insufficiency questionnaire [52]. Similar data were obtained by Cote and co-workers who investigated 683 stable COPD out-patients and found that anaemic patients showed a significantly higher modified Medical Research Council dyspnoea scale than non-anaemic patients [53]. In a post-hoc analysis of patients with COPD the physical function scores of the HRQOL questionnaire Short Form-36 were significantly lower in patients with anaemia compared to those without [54].

\section{Associations between mental health and functional data}

We found significant negative correlations between 6MWD values and several dimensions of mental health. The 6-MWD test is considered as an integrative measure influenced by physiological, physical and emotional aspects [11], and there is evidence that HRQOL increases with physical activity [55]. From this point of view, our findings regarding 6-MWD appear to be consistent with those of previous studies [56,57].

Anxiety showed a negative linear association with age. Cleland et al. found a similar association with significantly higher anxiety and depression levels in COPD patients below the age of 60 years [39]. This was interpreted on the one hand due to a better acceptance of symptoms in the elderly as a predictable 
Table 3 Spearman's rank correlation coefficient between biomarkers I

\begin{tabular}{|c|c|c|c|c|c|c|}
\hline & 25-OHD (nmol/l) & CRP (mg-dl) & $\operatorname{ESR}(\mathrm{mm} / \mathrm{h})$ & Leucocytes $\left(10^{3} / \mu \mathrm{l}\right)$ & $\mathrm{SA}-\mathrm{A}(\mathrm{mg} / \mathrm{l})$ & NT-proBNP (pg/ml) \\
\hline 25-OHD (nmol/l) & 1.00 & & & & & \\
\hline CRP (mg/dl) & 0.08 & 1.00 & & & & \\
\hline $\mathrm{ESR}(\mathrm{mm} / \mathrm{h})$ & 0.15 & 0.34 & 1.00 & & & \\
\hline Leucocytes $\left(10^{3} / \mu \mathrm{l}\right)$ & 0.15 & 0.18 & 0.07 & 1.00 & & \\
\hline SA-A (mg/l) & 0.17 & $0.512^{* *}$ & 0.22 & $0.593^{* *}$ & 1.00 & \\
\hline NT-proBNP (pg/ml) & 0.06 & 0.14 & -0.08 & 0.04 & 0.10 & 1.00 \\
\hline Troponin I (ng/ml) & 0.00 & 0.12 & 0.10 & 0.18 & 0.11 & $0.493^{* *}$ \\
\hline HbAlc (\%) & -0.01 & 0.28 & $0.415^{*}$ & 0.18 & 0.11 & 007 \\
\hline $\mathrm{Hb}(\mathrm{g} / \mathrm{dl})$ & -0.28 & -0.25 & $-0.360^{*}$ & 0.02 & -0.10 & -0.05 \\
\hline Hc (\%) & $-0.355^{*}$ & -0.19 & $-0.353^{*}$ & 0.06 & -0.12 & -0.04 \\
\hline Creatinine (mg/dl) & -0.26 & -0.07 & 0.11 & 0.04 & 0.00 & $0.355^{*}$ \\
\hline TSH (mU/l) & -0.06 & 0.29 & 0.20 & 0.05 & 0.04 & $0.316^{*}$ \\
\hline
\end{tabular}

${ }^{*} p=0.05 ;{ }^{* *} p=0.01$

25-OHD 25-OH-cholecalciferol, CRP C-reactive protein, ESR erythrocyte sedimentation rate, SA-A serum amyloid-A, NT-proBNP N-terminal pro-brain natriuretic peptide, $\mathrm{HbAlc}$ glycosylated haemoglobin, $\mathrm{Hb}$ haemoglobin, $\mathrm{Hc}$ haematocrit, $T S H$ thyroid-stimulating hormone

Highlighted in bold: $\mathrm{p} \leq 0.05$

late life stressor, and on the other hand as an indication that in contrast to healthy subjects of the same age younger COPD patients have to deal with functional and physical impairment and therefore suffer more psychologically [39].

\section{Survival}

Mental health in terms of PHQ-stress and systemic inflammation in terms of leucocyte levels were significantly related to 3-year survival in univariate survival analysis. Regarding the predictive value of leukocytes levels for long-term survival it appears reassuring that in the large Evaluation of COPD Longitudinally to Identify Predictive Surrogate Endpoints (ECLIPSE) study, comprising 1843 COPD patients, leukocyte numbers improved the predictive value of established risk factors [16]. In contrast, in the present investigation the level of serum CRP was not significantly linked to survival. This is in line with the results of the study of de Torres and co-workers who postulated that, particularly in patients with moderate to severe COPD, the magnitude of
CRP-levels could be critically influenced by other factors such as morbidity (cardiovascular disease, metabolic syndrome, hypertension etc.), degree of physical activity, diet, smoking status and medications [58]. Conversely, the prognostic value of CRP was higher in large epidemiological studies covering patients with less severe COPD [59].

In the present study, neither depression, nor anxiety has shown to be associated with survival. Other studies revealed depression as negative prognostic factor for mid- to long-term survival (1 to 3 years) for COPD patients being in a stable state of disease [6-8], and for short-term survival (6 months) after recovering from an acute exacerbation [60]. Possible reasons for this differences might be the use of different cut-off values, questionnaires and observation time (Beck Depression Inventory: cut-off $\geq 19$ [8], quintile of $\geq 15$ compared with $<5$ [7]; Yesavage depression score: cut-off 5 and 11 [6]; HADS-A/D: cut-off $\geq 8$ [60]). Furthermore, anxiety was also no significant predictor of mid- to long-term survival [7], but marginal significant after acute exacerbation [60].

Table 4 Spearman's rank correlation coefficient between biomarkers ॥

\begin{tabular}{|c|c|c|c|c|c|c|}
\hline & Troponin I (ng/ml) & HbAlc (\%) & $\mathrm{Hb}(\mathrm{g} / \mathrm{dl})$ & $\mathrm{Hc}(\%)$ & Creatinine (mg/dl) & $\mathrm{TSH}(\mathrm{mU} / \mathrm{l})$ \\
\hline Troponin I (ng/ml) & 1.00 & & & & & \\
\hline HbAlc (\%) & 0.22 & 1.00 & & & & \\
\hline $\mathrm{Hb}(\mathrm{g} / \mathrm{dl})$ & 0.12 & 0.24 & 1.00 & & & \\
\hline Hc (\%) & 0.19 & 0.27 & $0.946^{*}$ & 1.00 & & \\
\hline Creatinine (mg/dl) & $0.432^{*}$ & 0.25 & 0.07 & 0.13 & 1.00 & \\
\hline TSH (mU/l) & -0.01 & 0.08 & -0.19 & -0.20 & 0.06 & 1.00 \\
\hline
\end{tabular}


Table 5 Linear univariate regression analysis of the questionnaire dimensions

\begin{tabular}{|c|c|c|}
\hline Explanatory Variable & B Slope (95 \% Cl) & $p^{*}$ \\
\hline \multicolumn{3}{|l|}{ Univariate analyses } \\
\hline \multicolumn{3}{|l|}{ HADS anxiety } \\
\hline Age (years) & $-0.11(-0.217-(-0.002))$ & 0.045 \\
\hline VC (\%) & $-0.06(-0.104-(-0.015))$ & 0.01 \\
\hline $\mathrm{FEV}_{1}(\%)$ & $-0.1(-0.174-(-0.02))$ & 0.015 \\
\hline 6-MWD (\%) & $-0.09(-0.136-(-0.035))$ & 0.002 \\
\hline $\mathrm{RV} / \mathrm{TLC}$ & $0.042(0.012-0.072)$ & 0.007 \\
\hline \multicolumn{3}{|l|}{ HADS depression } \\
\hline $\mathrm{Hb}(\mathrm{g} / \mathrm{dl})$ & $-0.94(-1.838-(-0.041))$ & 0.041 \\
\hline Hc (\%) & $-0.3(-0.577-(-0.029))$ & 0.031 \\
\hline 6-MWD (\%) & $-0.12(-0.234-(-0.012))$ & 0.031 \\
\hline \multicolumn{3}{|l|}{ PHQ-15 (somatisation) } \\
\hline 6-MWD (\%) & $-0.15(-0.276-(-0.025))$ & 0.021 \\
\hline \multicolumn{3}{|l|}{ PHQ-stress } \\
\hline Leucocytes $\left(10^{3} / \mu \mathrm{l}\right)$ & $0.4(0.013-0.777)$ & 0.043 \\
\hline \multicolumn{3}{|l|}{ SGRQ-total } \\
\hline 6-MWD & $-0.44(-0.714-(-0.166))$ & 0.003 \\
\hline \multicolumn{3}{|l|}{ STAl-state anxiety } \\
\hline VC (\%) & $-0.23(-0.453-(-0.01))$ & 0.041 \\
\hline \multicolumn{3}{|l|}{ STAI-trait anxiety } \\
\hline Leucocytes $\left(10^{3} / \mu \mathrm{l}\right)$ & $1.1(0.1117-2.089)$ & 0.03 \\
\hline SA-A (mg/l) & $0.17(0.055-0.276)$ & 0.005 \\
\hline \multicolumn{3}{|c|}{$\begin{array}{l}\text { *Only the significant }(p \leq 0.05) \text { variables are shown. The questionnaire } \\
\text { dimensions were the dependent variables } \\
\text { 6-MWD, six-minute walk test, ESR erythrocyte sedimentation rate, FEV1, forced } \\
\text { expiratory volume in } 1 \mathrm{~s}, H b \text { haemoglobin, Hc haematocrit, SA-A serum } \\
\text { amyloid-A, } R V \text { residual volume, } T L C \text { total lung capacity, VC vital capacity, HADS } \\
\text { Hospital Anxiety and Depression Scale, PHQ Patient Health Questionnaire, SGRQ } \\
\text { St. George's Respiratory Questinnaire, STAI State-trait anxiety inventory }\end{array}$} \\
\hline
\end{tabular}

To the best of our knowledge, this is the first study to investigate the PHQ-stress score and survival in patients with COPD. Psychosocial stress occurs when the individual recognises that environmental demands strain or exceed their adaptive capability. Chronic psychosocial stress in particular seems to influence the course of diseases, including cardiovascular disease, upper respiratory infections, autoimmune diseases, diabetes and depression [61]. This is in line with our findings of an association of increased psychosocial stress levels and mortality in severe COPD. Moreover, sleep is a vital counterbalance to stress enabling the body to recover and is essential for coping with stress [62]. Therefore, a disease such as COPD, with its high prevalence of sleep disturbance, is at risk of causing increased stress. Chronic psychosocial stress also can lead to glucocorticoid receptor resistance and consequently interferes with the control of inflammation [63], which could also explain its positive linear correlation with leucocyte numbers.
Table 6 Multivariate linear regression analysis of the questionnaire dimensios

\begin{tabular}{llll}
\hline Explanatory Variable & Slope $(95 \% \mathrm{Cl})$ & $p$ & Model R \\
\hline HADS Anxiety & & & \\
RV/TLC & $0.02(-0.017-0.056)$ & 0.272 & \\
6-MWD (\%) & $-0.072(-0.128-(-0.016))$ & $\mathbf{0 . 0 1 4}$ & 0.401 \\
VC (\%) & $-0.027(-0.090-0.037)$ & 0.391 & \\
6-MWD (\%) & $-0.078(-0.132-(-0.024))$ & $\mathbf{0 . 0 2 4}$ & 0.394 \\
FEV $1 \%$ (\%) & $-0.079(-0.124-0.065)$ & 0.519 & \\
6-MWD (\%) & $-0.030(-0.135-(-0.024))$ & $\mathbf{0 . 0 0 8}$ & 0.384 \\
HADS Depression & & & \\
6-MWD (\%) & $-0.111(-0.227-0.005)$ & 0.059 & \\
HC (\%) & $-0.168(-0.600-0.265)$ & 0.429 & 0.228 \\
6-MWD (\%) & $-0.111(-0.228-(-0.005))$ & $\mathbf{0 . 0 4 2}$ & \\
Hb (g/dl) & $-0.635(-1.917-0.646)$ & 0.314 & 0.243 \\
\hline
\end{tabular}

25-OHD 25-OH-cholecalciferol, 6-MWD six-minute walk test, ESR erythrocyte sedimentation rate, $F E V 1$, forced expiratory volume in $1 \mathrm{~s}, \mathrm{Hb}$ haemoglobin, $\mathrm{Hc}$ haematocrit, $R V$ residual volume, $S A-A$ serum amyloid-A, TLC total lung capacity, VC vital capacity, HADS Hospital Anxiety and Depression Scale, STAI State-trait anxiety inventory. Questionnaire dimensions were the dependent variables Highlighted in bold: $\mathrm{p} \leq 0.05$

Table 7 Cox regression analysis of survival according to categorisation of variables < vs. $>$ the median value

\begin{tabular}{lll}
\hline Explanatory Variable & $\mathrm{HR}(95 \% \mathrm{Cl})$ & ${ }^{*} p$ \\
\hline Univariate analysis & & \\
PHQ-stress & $4.92(1.06-22.85)$ & $\mathbf{0 . 0 4 2}$ \\
Leucocytes & $2.97(1.06-8.36)$ & $\mathbf{0 . 0 3 8}$ \\
6-MWD (\%) & $2.07(0.6-7.07)$ & 0.248 \\
BMl & $0.77(0.30-1.95)$ & 0.58 \\
FEV $_{1}, \%$ predicted & $1.51(0.59-3.89)$ & 0.395
\end{tabular}

Multivariate analyses

Model I

$\begin{array}{lll}\text { 6-MWD (\%) } & 19.58(1.01-380.75) & \mathbf{0 . 0 4 9} \\ \text { BMI } & 48.73(1.21-1969.65) & \mathbf{0 . 0 3 9} \\ \text { FEV } 1, \% \text { predicted } & 3.37(0.35-32.55) & 0.295 \\ \text { PHQ-stress } & 45.63(1.72-1,208.48) & \mathbf{0 . 0 2 2}\end{array}$

Model 2

\begin{tabular}{lll} 
6-MWD (\%) & $2.6(0.71-9.57)$ & 0.151 \\
BMI & $1.34(0.38-4.79)$ & 0.650 \\
FEV $_{1}, \%$ predicted & $1.03(0.25-4.18)$ & 0.970 \\
Leucocytes & $3.41(0.91-12.85)$ & 0.069 \\
\hline
\end{tabular}

*Only the significant $(p \leq 0.05)$ univariate variables for physical function, demographic data and biomarkers are shown. Model 1 and 2: Cox proportional hazard model (method: stepwise backward likelihood ratio) including known risk factors for survival in COPD (6-MWD, body mass index, $\mathrm{FEV}_{1}$, (\% predicted)) as well as PHQ-stress (model 1) and leucocytes (model 2) PHQ Patient Health Questionnaire, \&-MWD six-minute walk test, BMI body mass index, FEV1 forced expiratory volume in $1 \mathrm{~s}$ Highlighted in bold: $\mathrm{p} \leq 0.05$ 

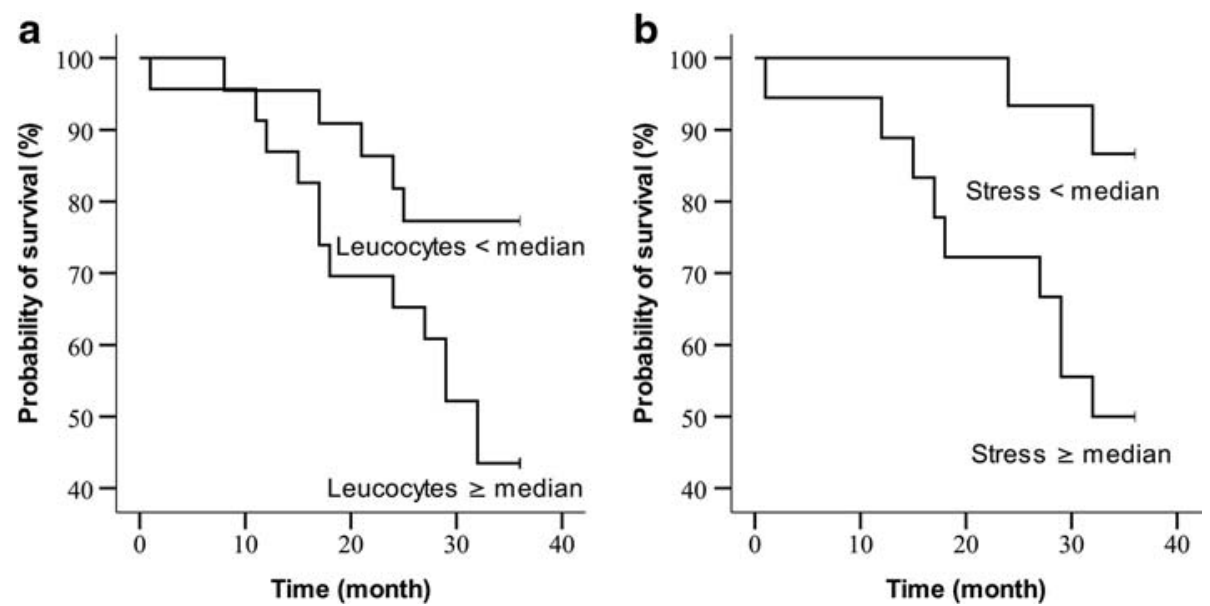

Fig. 4 Kaplan Meier charts for leucocyte numbers (a) and PHQ-stress (b) using the respective median values as cut-off (leucocytes: median $=9.7$ $10^{3} / \mu \mathrm{l}, p=0.028 ; \mathrm{PHQ}-\mathrm{stress}$ : median $=6, p=0.023$ ). PHQ, Patient Health Questionnaire; SGRQ, St. George's Respiratory

Questionnaire; STAI, State-Trait Anxiety Inventory

\section{Limitations}

Firstly, the small sample size allowed detecting only relatively strong associations, and limited statistical power especially for multivariate analyses. Secondly, the patients' motivation to start and to complete the questionnaires apparently dwindled with the number of pages. Fewer questionnaires would have increased motivation and compliance. Thirdly, not all questionnaires have an accepted score classification; therefore, the use of median values was the simplest way for comparison. It is, therefore, possible that associations would be different using other classification systems. Fourthly, corticosteroids (systemic and/or inhaled) and statins might influence especially inflammation markers like CRP, leucocyte numbers and SA-A; osteoporosis prophylaxis might affect the level of 25-OHD, and anxiolytics, antidepressants, morphine/morphine derivates, corticosteroids and theophylline the questionnaire results. Finally, the seemingly paradoxical tendencies regarding survival and lung function or 6-MWD suggest that we had to deal with a specific selection of patients. In view of this it seems of even greater interest that psychosocial stress was a predictor for survival even in patients with a tendency towards prolonged survival despite worse lung function or 6-MWD.

\section{Conclusion}

In conclusion, the different dimensions of mental health assessed in this pilot study were related to serum biomarkers although these associations were rather weak. This might be indicative of the heterogeneity of the disease. Regarding the predictive value for long-term survival, the PHQ-stress score was an independent prognostic factor in multivariate analyses, whereas among biomarkers, only leukocyte numbers showed an association and only in univariate analysis. The observation that psychosocial stress had a greater prognostic value than conventional biomarkers suggests that this easily measured parameter would be useful in the prognostic assessment of patients with severe COPD and - with regard to the multiple systemic effects - probably should be included in the assessment of COPD.

This pilot study may help to design further larger prospective trials to investigate the associations between mental health, serum biomarkers and survival in severe COPD in more detail.

\section{Competing interests}

None of the authors have any conflicting interests to declare.

\section{Authors' contributions}

CZ conducted data collection, performed data analysis and drafted the manuscript. SHB conducted data collection and contributed to the manuscript. MP reviewed and contributed to the manuscript. RJ helped with data analysis, reviewed the manuscript and contributed to critical revision of the manuscript. SB conceived the study, helped with data analysis, coordination and drafting of the manuscript. All authors read and approved the final manuscript.

\section{Acknowledgements}

The work for this study was performed at the Center for Pneumology at Donaustauf Hospital.

\section{Author details}

${ }^{1}$ Department of Internal Medicine, HELIOS Hospital Munich-Perlach, Munich, Germany. ${ }^{2}$ Center for Pneumology, Donaustauf Hospital, Donaustauf, Germany. ${ }^{3}$ Institute and Outpatient Clinic for Occupational, Social and Environmental Medicine, Ludwig-Maximilian University, Munich, Germany. ${ }^{4}$ Department of Internal Medicine II, Division of Respirology, University of Regensburg, Regensburg, Germany. ${ }^{5}$ Department of Internal Medicine III, RoMed Clinical Center Rosenheim, Rosenheim, Germany.

Received: 9 November 2015 Accepted: 5 January 2016

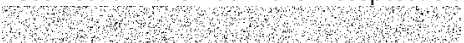

\section{References}

1. Woodruff PG, Agusti A, Roche N, Singh D, Martinez FJ. Current concepts in targeting chronic obstructive pulmonary disease pharmacotherapy: making progress towards personalised management. Lancet. 2015;385(9979):1789-98. 
2. Di Marco F, Verga M, Reggente M, Maria Casanova F, Santus P, Blasi F, et al. Anxiety and depression in COPD patients: The roles of gender and disease severity. Respir Med. 2006;100(10):1767-74

3. Goodwin RD, Chuang S, Simuro N, Davies M, Pine DS. Association between Lung Function and Mental Health Problems among Adults in the United States: Findings from the First National Health and Nutrition Examination Survey. Am J Epidemiol. 2006;165(4):383-8.

4. Karajgi B, Rifkin A, Doddi S, Kolli R. The prevalence of anxiety disorders in patients with chronic obstructive pulmonary disease. Am J Psychiatry. 1990;147(2):200-1.

5. Yellowlees PM, Alpers JH, Bowden JJ, Bryant GD, Ruffin RE. Psychiatric morbidity in patients with chronic airflow obstruction. Med J Aust. 1987;146(6):305-7.

6. Almagro P, Calbo E, Ochoa de Echagüen A, Barreiro B, Quintana S, Heredia JL, et al. Mortality after hospitalization for COPD. Chest. 2002;121(5):1441-8.

7. Fan VS. Sex, Depression, and Risk of Hospitalization and Mortality in Chronic Obstructive Pulmonary Disease. Arch Intern Med. 2007;167(21):2345.

8. de Voogd JN. Depressive Symptoms as Predictors of Mortality in Patients With COPD. Chest. 2009;135(3):619.

9. CDC - Health-Related Quality of Life - HRQOL. http://www.cdc.gov/hrqol/. Accessed 7 Dec 2014

10. Budweiser S, Hitzl AP, Jörres RA, Schmidbauer K, Heinemann F, Pfeifer M. Health-related quality of life and long-term prognosis in chronic hypercapnic respiratory failure: a prospective survival analysis. Respir Res. 2007;8(1):92.

11. Engström CP, PLLSSM. Health-related quality of life in COPD: why both disease-specific and generic measures should be used. Eur Respir J. 2001;18(1):69-76.

12. Seemungal TA. DGPEBJJDWJ Effect of exacerbation on quality of life in patients with chronic obstructive pulmonary disease. Am J Respir Crit Care Med. 1998;157:1418-22.

13. Lacoma A, Prat C, Andreo F, Dominguez J. Biomarkers in the management of COPD. Eur Respir Rev. 2009;18(112):96-104.

14. Dominguez J, Lacoma A, Prat C, Andreo F, Lores L, Ruiz-Manzano J, Ausina $\checkmark$. Value of procalcitonin, $C$-reactive protein, and neopterin in exacerbations of chronic obstructive pulmonary disease. Int J Chron Obstruct Pulmon Dis. 2011;6:157-169.

15. Chang CL, Robinson SC, Mills GD, Sullivan GD, Karalus NC, McLachlan JD, et al. Biochemical markers of cardiac dysfunction predict mortality in acute exacerbations of COPD. Thorax. 2011;66(9):764-8.

16. Celli BR, Locantore N, Yates J, Tal-Singer R, Miller BE, Bakke P, et al. Inflammatory Biomarkers Improve Clinical Prediction of Mortality in Chronic Obstructive Pulmonary Disease. Am J Respir Crit Care Med. 2012;185(10):1065-72.

17. Pascoe S, Locantore N, Dransfield MT, Barnes NC, Pavord ID. Blood eosinophil counts, exacerbations, and response to the addition of inhaled fluticasone furoate to vilanterol in patients with chronic obstructive pulmonary disease: a secondary analysis of data from two parallel randomised controlled trials. Lancet Respir Med. 2015;3(6):435-42.

18. Howren MB, Lamkin DM, Suls J. Associations of Depression With C-Reactive Protein, IL-1, and IL-6: A Meta-Analysis. Psychosom Med. 2009;71(2):171-86.

19. Alves de Rezende $\mathrm{CH}$, Coelho LM, Oliveira LM, Penha Silva N. Dependence of the geriatric depression scores on age, nutritional status, and haematologic variables in elderly institutionalized patients. J Nutr Health Aging. 2009;13(7):617-21.

20. Chueire VB, Silva ETB, Perotta E, Romaldini JH, Ward LS. High serum TSH levels are associated with depression in the elderly. Arch Gerontol Geriatr. 2003;36(3):281-8.

21. Saxena J, Singh PN, Srivastava U, Siddiqui AQ. A study of thyroid hormones (t(3), t(4) \& tsh) in patients of depression. Indian J Psychiatry. 2000;42(3):243-6.

22. Ganji V, Milone C, Cody MM, McCarty F, Wang YT. Serum vitamin D concentrations are related to depression in young adult US population: the Third National Health and Nutrition Examination Survey. Int Arch Med. 2010;3(1):29.

23. Hoogendijk WJG. Depression Is Associated With Decreased 25Hydroxyvitamin D and Increased Parathyroid Hormone Levels in Older Adults. Arch Gen Psychiatry. 2008;65(5):508.

24. Jorde R, Sneve M, Figenschau Y, Svartberg J, Waterloo K. Effects of vitamin $D$ supplementation on symptoms of depression in overweight and obese subjects: randomized double blind trial. J Intern Med. 2008;264(6):599-609.

25. Jorde R, Waterloo K, Saleh F, Haug E, Svartberg J. Neuropsychological function in relation to serum parathyroid hormone and serum $25-$ hydroxyvitamin D levels. The Tromso study. J Neurol. 2006;253(4):464-70.
26. Standardization of spirometry-1987 update. Official statement of American Thoracic Society. Respir Care. 1987:32(11): 1039-1060. http://www.ncbi.nlm. nih.gov/pubmed/10315742?dopt=Abstract\&holding=f1000,f1000m,isrctn

27. Cotes JE, Chinn DJ, Quanjer PH, Roca J, Yernault JC. Standardization of the measurement of transfer factor (diffusing capacity). Report Working Party Standardization of Lung Function Tests, European Community for Steel and Coal. Official Statement of the European Respiratory Society. Eur Respir J Suppl. 1993;16:41-52.

28. ATS statement: guidelines for the six-minute walk test. Am J Respir Crit Care Med. 2002:166(1):111-117. http://www.ncbi.nlm.nih.gov/pubmed/12091180

29. Enright $P L$, Sherrill $D L$. Reference equations for the six-minute walk in healthy adults. Am J Respir Crit Care Med. 1998;158(5 Pt 1):1384-7.

30. Herrmann-Lingen C, Buss U, Snaith RP. Hospital anxiety and depression scale. HADS-D; deutsche Version. 3rd ed. Bern: Huber; 2011.

31. Spitzer RL, Kroenke K, Williams JB. Validation and utility of a self-report version of PRIME-MD: the PHQ primary care study. Primary Care Evaluation of Mental Disorders. Patient Health Questionnaire. JAMA. 1999;282(18):1737-44.

32. Jones PW, QFBCLP. A self-complete measure of health status for chronic airflow limitation. The St. George's Respiratory Questionnaire. Am Rev Respir Dis. 1992;145:1321-7.

33. Laux L. Das State-Trait-Angstinventar. Beltz, Göttingen: STAl. Beltz-Test; 1981.

34. Zigmond AS, Snaith RP. The hospital anxiety and depression scale. Acta Psychiatr Scand. 1983;67(6):361-70.

35. Löwe BSR, Zipfel S, Herzog W. Gesundheitsfragebogen für Patienten (PHQ D). Komplettversion und Kurzform. Testmappe mit Manual, Fragebögen, Schablonen 2. Auflage. Karlsruhe: Pfizer; 2002.

36. Kroenke K, Spitzer RL, Williams JB. The PHQ-9: validity of a brief depression severity measure. J Gen Intern Med. 2001;16(9):606-13.

37. Kroenke K, Spitzer RL, Williams JBW. The PHQ-15: validity of a new measure for evaluating the severity of somatic symptoms. Psychosom Med. 2002; 64(2):258-266

38. van Manen JG. Risk of depression in patients with chronic obstructive pulmonary disease and its determinants. Thorax. 2002;57(5):412-6.

39. Cleland JA, Lee AJ, Hall S. Associations of depression and anxiety with gender, age, health-related quality of life and symptoms in primary care COPD patients. Fam Pract. 2007;24(3):217-23.

40. Kocalevent R, Hinz A, Brähler E. Standardization of a screening instrument (PHQ-15) for somatization syndromes in the general population. BMC Psychiatry. 2013;13(1):91.

41. Klapow J, Kroenke K, Horton T, Schmidt S, Spitzer R, Williams JBW. Psychological disorders and distress in older primary care patients: a comparison of older and younger samples. Psychosom Med. 2002:64(4):635-43.

42. Justo D, Arbel Y, Altberg G, Kinori M, Shirom A, Melamed S, et al. Inflammation Markers in Individuals with History of Mental Health Crisis. Inflammation. 2008:31(4):254-9.

43. Miller AH, Maletic V, Raison CL. Inflammation and Its Discontents: The Role of Cytokines in the Pathophysiology of Major Depression. Biol Psychiatry. 2009;65(9):732-41.

44. Bankier B, Barajas J, Martinez-Rumayor A, Januzzi JL. Association between C-reactive protein and generalized anxiety disorder in stable coronary heart disease patients. Eur Heart J. 2008;29(18):2212-7.

45. Pitsavos C, Panagiotakos DB, Papageorgiou C, Tsetsekou E, Soldatos C, Stefanadis C. Anxiety in relation to inflammation and coagulation markers, among healthy adults: The ATTICA Study. Atherosclerosis. 2006;185(2):320-6.

46. Thomsen M, Dahl M, Lange P, Vestbo J, Nordestgaard BG. Inflammatory Biomarkers and Comorbidities in Chronic Obstructive Pulmonary Disease. Am J Respir Crit Care Med. 2012;186(10):982-8.

47. Block ML, Zecca L, Hong J. Microglia-mediated neurotoxicity: uncovering the molecular mechanisms. Nat Rev Neurosci. 2007;8(1):57-69.

48. Pluchino S, Muzio L, Imitola J, Deleidi M, Alfaro-Cervello C, Salani G, et al. Persistent inflammation alters the function of the endogenous brain stem cell compartment. Brain. 2008;131(10):2564-78.

49. Al-shair K, Kolsum U, Dockry R, Morris J, Singh D, Vestbo J. Biomarkers of systemic inflammation and depression and fatigue in moderate clinically stable COPD. Respir Res. 2011;12(1):3.

50. Sapey E. COPD exacerbations \{middle dot\} 2: Aetiology. Thorax. 2006: 61(3):250-8.

51. Bozinovski S, Hutchinson A, Thompson M, MacGregor L, Black J, Giannakis E, et al. Serum Amyloid A Is a Biomarker of Acute Exacerbations of 
Chronic Obstructive Pulmonary Disease. Am J Respir Crit Care Med. 2008;177(3):269-78.

52. Schneckenpointner R, Jörres RA, Meidenbauer N, Kollert F, Pfeifer M, Budweiser $\mathrm{S}$. The clinical significance of anaemia and disturbed iron homeostasis in chronic respiratory failure. Int J Clin Pract. 2014;68(1):130-8.

53. Cote C, Zilberberg MD, Mody SH, Dordelly L, Celli B. Haemoglobin level and its clinical impact in a cohort of patients with COPD. Eur Respir J. 2007;29(5):923-9.

54. Krishnan G, GBMPMAOHFJTMSH. Association between anemia and quality of life in a population sample of individuals with chronic obstructive pulmonary disease. BMC Pulmonary Medicine. 2006;6:23.

55. Esteban C, Quintana JM, Aburto M, Moraza J, Egurrola M, Perez-Izquierdo J, et al. Impact of changes in physical activity on health-related quality of life among patients with COPD. Eur Respir J. 2010;36(2):292-300.

56. Pinto-Plata V, Cote C, Cabral H, Taylor J, Celli B. The 6-min walk distance: change over time and value as a predictor of survival in severe COPD. Eur Respir J. 2004;23(1):28-33.

57. Waschki B. Physical Activity Is the Strongest Predictor of All-Cause Mortality in Patients With COPD. Chest. 2011;140(2):331.

58. de Torres JP. C-Reactive Protein Levels and Survival in Patients With Moderate to Very Severe COPD*. Chest. 2008;133(6):1336.

59. Dahl M, Vestbo J, Lange P, Bojesen SE, Tybjærg-Hansen A, Nordestgaard BG. C-reactive Protein As a Predictor of Prognosis in Chronic Obstructive Pulmonary Disease. Am J Respir Crit Care Med. 2007;175(3):250-5.

60. Ng T. Depressive Symptoms and Chronic Obstructive Pulmonary Disease. Arch Intern Med. 2007;167(1):60.

61. Cohen S, Janicki-Deverts D, Miller GE. Psychological Stress and Disease. JAMA. 2007;298(14):1685

62. Danielsson M, Heimerson I, Lundberg U, Perski A, Stefansson C, Akerstedt T. Psychosocial stress and health problems: Health in Sweden: The National Public Health Report 2012. Chapter 6. Scand J Public Health. 2012;40(9 Suppl):121-34.

63. Cohen S, Janicki-Deverts D, Doyle WJ, Miller GE, Frank E, Rabin BS, et al. Chronic stress, glucocorticoid receptor resistance, inflammation, and disease risk. Proc Natl Acad Sci. 2012;109(16):5995-9.

\section{Submit your next manuscript to BioMed Central and we will help you at every step:}

- We accept pre-submission inquiries

- Our selector tool helps you to find the most relevant journal

- We provide round the clock customer support

- Convenient online submission

- Thorough peer review

- Inclusion in PubMed and all major indexing services

- Maximum visibility for your research

Submit your manuscript at www.biomedcentral.com/submit 